Accelerator Development Department

$\mathrm{AD} / \mathrm{RHIC}-50$ BROOKHAVEN NATIONAL LABORATORY

Associated Universities, Inc.

Upton, New York 11973

RHIC TeChnical Note No. 50

The Heavy Ion Injection Scheme for RHIC

M. Rhoades-Brown

$3 / 20 / 89$ 
Particle Accelerator Conference, Chicago, IL, March 20-23, 1989

THE HEAVY ION INIECTION SCHEME FOR RHIC*

\author{
MJ. Rhoades-Brown \\ Brookhaven National Laboratory \\ Upton, New York 11973
}

\begin{abstract}
The Relativistic Heavy lon Collider (RHIC) at Brookhaven has a multi-component injection system. The Collider requires very heavy ions such as ${ }_{79}^{19}$ Au to be injected fully stripped of atomic electrons, at a kinetic energy of approximately $10 \mathrm{GeV} /$ aucleon. However, the heavy ions are produced initially at a negative ion source and accelerated first in a $15 \mathrm{MV}$ Tandem. These partially stripped ions have a kinetic energy of approximately $1 \mathrm{MeV} /$ nucleon on leaving the Tandem. In order to achieve the injection requirements for RHIC, the partially stripped ions are accelerated in the Booster (currently under construction) and pass through a stripping foil on their way to the Alternating Gradient Synchrotron (AGS), where they are further accelerated before injection into RHIC. Recent theoretical calculations have shown quite convincingly that very heavy ions with 2 electrons in the filled $\mathrm{K}$-shell may be accelerated with negligible loss in the AGS.
\end{abstract}

\section{Description of the Facility}

\subsection{Collider Performance}

The complete RHIC facility will be a complex set of accelerators and beam transfer equipmment connecting them. ${ }^{1}$ The final accelerator, the collider itself, has a circumference of $3883.8 \mathrm{~m}$, and a magnetic field generated by superconducting magnets of about 3 . $45 \mathrm{~T}$. With these parameters, the maximum energy is $-100 \mathrm{GeV} / \mathrm{u}$ for gold ions. The design specifications of RHIC call for $-1 \times 10^{9}$ ions per bunch for gold or $-6 \times 10^{10}$ ions in 57 bunches in each ring of the collider. 'For head-on collisions with gold beams at top energy, a luminosity of $-2 \times 10^{26} \mathrm{~cm}^{2} \mathrm{sec}^{-1}$ averaged over a $10 \mathrm{~h}$ beam lifetime is expected.

For heavy ion injection into RHIC, the rigidity values of the collider at injection ( $B P=96.7 \mathrm{~T} \cdot \mathrm{m})$, and the desired beam lifetime of 10 hours within RHIC demand that fully stripped ions be injected at kinetic energies in excess of $10 \mathrm{GeV} / \mathrm{u}$.

\section{RHIC Injector Complex}

\section{II.1 Overview}

The existing accelerator complex at Brookhaven (Figure 1) consisting of the Tandem Van de Graaff, the Heavy Ion Transfer line, the Alternating Gradient Synchrotron and the Booster Synchrotron will serve as the injector of heavy ions for RHIC. Operation of the AGS for heavy ion experiments started in October 1986 with the delivery of $0^{8+}$ beams. Completion of the AGS booster synchrotron in 1991 will extend the mass range to the heaviest ions, typically ${ }^{179} \mathrm{Au}$, with ${ }^{238} \mathrm{U}$ a definite possibility. In this paper, the multi-component injection mode of the collider will be discussed with special emphasis on the expected particle intensities.

The atomic electrons are stripped by passing the ions through foils $S_{T}, S_{B}$ and $S_{A}$ at various stages of acceleration. The location of these foils is shown in Fig. 2. After passage through the foils, a broad distribution of charge states results. Normally only the most probable charge state is accelerated at the next stage.

\footnotetext{
*Work performed under the auspices of the U.S. Deparment of Energy
}

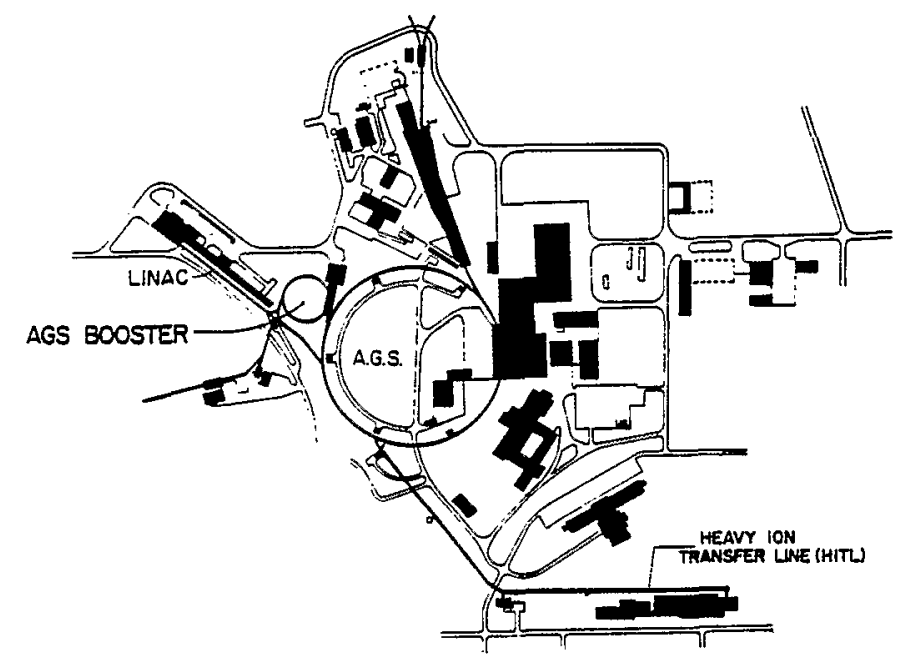

Fig. 1. Overview of RHIC Injector Complex

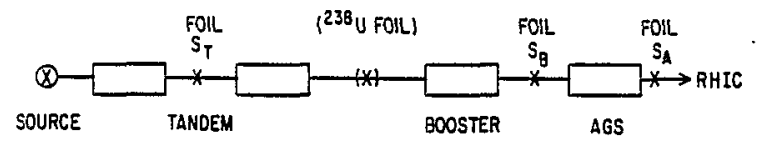

Fig. 2. Location of Stripping Foils

The design specifications of RHIC call for $10^{9}$ particles per bunch of fully stripped ${ }^{197} \mathrm{Au}$ ions. In order to achieve this value, several limiting characteristics of the injection components must be respected. ${ }^{2}$ These include the vacuum of the AGS $\left(10^{-7}\right.$ Torr at present, $10^{-8}$ Torr after upgrade program), which prohibits the acceleration of heavy ions with many atomic electrons, the lowest achievable frequency $(215 \mathrm{kHz})$ of the Booster cavities, the stripping foil efficiencies, and the current technological limitations of heavy ion sources at the Tandem. Respecting these constraints, it is possible to achieve $10^{9}$ particles per bunch for ${ }^{197} \mathrm{Au}$. However, the wide variation of atomic structure across the periodic table prevents a simple uniform injection scheme for all heavy ion species.

\section{I1.2 The Tandem Van de Graaf}

The acceleration of heavy ion beams will be started using one of two existing Tandem Van de Graaff accelerators. The construction of a transfer line from the Tandem to the AGS, located in a 
$1800 \mathrm{ft}$ long tunnel, was completed in 1986. Also shown in Fig. 1 is the extesded heavy ion transfer line which will enable heavy ions to be injected into the Booster.

Up to $240 \mu \mathrm{A}$ of usable ${ }^{\text {in }}$ Au are now reliably available at the

Tandem source. ${ }^{3}$ More recently, a new experimental source has been inveatigated at Brookhuven, for which currents of up to $10 \mathrm{~mA}$ of ${ }^{197}$ Au have been claimed elsewhere. ${ }^{4}$ It remains to be seen how much of this current is available for injection into the small Tandem aperture.

Table II-1 shows the Tandem operation parameters for a bigh voliage terminal potential of $15 \mathrm{MV}, \mathrm{S}_{\mathrm{T}}$ and $\mathrm{Q}_{\mathrm{T}}$ are the stripping efficiency and moat probable charge state after the negative ions have been accelerated to the high voltage terminal and stripped in the thin carbon foil inside the Tandem. The ions leave the accelerator with an energy $\left(1+Q_{T}\right) \times 15$ MV. For Table II-1, a source current of $200 \mu \mathrm{A}$ and a pulse length of $110 \mu \mathrm{s}$ was assumed. The transmission efficiency for the Tandem was taken to be 75\%.

Uranium is shown as a viable species because of recent calculations showing that it is possible to accelerate ${ }^{233} \mathrm{U}$ in a charge $90^{+}$ state within the AGS. ${ }^{3}$ In the future, this nucleus will be of great interest for both fixed target (AGS) and RHIC operation because of the enormous electric fields generated in nuclear collisions. It is noted however, that at the present time an adequate source for Uranium does not exist at Brookhaven.

Table L-1. Tandem Operation Parameters

\begin{tabular}{lccccc}
\hline & \multicolumn{5}{c}{ Kin. Energy } \\
& $Q_{T}$ & $S_{\mathrm{T}}$ & MeV/u & $\beta$ & Ions/Puise* \\
\hline Deuterium & +1 & $70 \%$ & 15.0 & 0.177 & $7.2 \times 10^{10}$ \\
Oxygen & +6 & $39 \%$ & 6.56 & 0.118 & $4.0 \times 10^{10}$ \\
Silicon & +9 & $30 \%$ & 5.36 & 0.107 & $3.1 \times 10^{10}$ \\
Copper & +11 & $27 \%$ & 2.86 & 0.078 & $2.8 \times 10^{10}$ \\
Iodine & +13 & $20 \%$ & 1.65 & 0.059 & $2.1 \times 10^{10}$ \\
Gold $^{\dagger}$ & +14 & $12 \%$ & 1.07 & 0.048 & $1.2 \times 10^{10}$ \\
Uranium $\S$ & +35 & $3.4 \%$ & 0.882 & 0.043 & $3.6 \times 10^{9}$ \\
\hline
\end{tabular}

$75 \%$ transmission efficiency, $200 \mu \mathrm{A}$ source current, $110 \mu$ pulse length. 'Equilibrium Charge State $13^{+}$.

SRequires Source Development and additional stripping foil at Tandem exit.

In order to accommodate Uranium in the Booster and AGS, a second stripping foil will be needed between the Tandem and Booster. This foil will produce Uranium in an equilibrium charge state of $35^{+}$, and will deplete the number of ions per Tandem pulse to $3.6 \times 10^{9}$ (stripping foil efficiency $=17 \%$ ).

\section{II.3 The Booster as Preinjector to RHIC}

This synchrotron will be commissioned in the summer of 1991 for heavy ions.

The extra kinetic energy provided by the Booster will enable additional atomic electrons to be stripped when passing through foil $S_{B}$ (see Fig. 2) situated between Booster and AGS. Hence, nuclei heavier than ${ }^{28} \mathrm{Si}$ will survive acceleration in the AGS vacuum, and not be lost to electron capture or stripping reactions. The Booster vacuum of $10^{-10}$ Torr seems adequate for the survival of essentially all the heavy ion beam during the acceleration cycle.

The beam is injected horizontally into the Booster, and stacked in betatron phase space by filling the machine circumference with the Tandem pulse for some number of consecutive turns. The betatron acceptance is assumed to be $50 \pi \mathrm{mm} \cdot \mathrm{mrad}$ in both planes.

The beam is captured at injection by an if system of harmonic number $h=2$ for light and medium mass ions, and with $h=3$ for heavy ions such as Au. ${ }^{6}$ After injection is completed, the if voltage is adiabatically increased to produce a bucket area of $\sim 0.07 \mathrm{eV} \cdot \mathrm{sec} /$ u. Theoretical studies show that if the rf bucket grows to full size in $10 \mathrm{msec}$ (approximately 10 synchrotron oscillation periods), $97 \%$ of the beam can be captured. 5 .6 Following capture, the bunched beam is accelerated. The three particle bunches are ejected simultaneously (within one revolution) from the Booster.
In Table II-3, the expected particle numbers per bunch in the Booster are shown logether with the if frequency ranges, and the normalized emittance. The same Tandem pulse length and source current as in Table II-1 were used here. In Table II-3, the particle numbers per bunch were calculated assuming an eight turn injection of $100 \%$ efficiency.

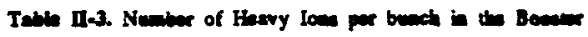

\begin{tabular}{|c|c|c|c|c|c|c|c|c|}
\hline Species: & $d$ & ${ }^{16} 0$ & ${ }^{28} \mathrm{Si}$ & ${ }^{63} \mathrm{C}$ & 127 I & ${ }^{197}$ Aw & ${ }^{238} \mathrm{v}$ & Uaie \\
\hline Hamonic, $h$ & 1 & 2 & 2 & 2 & 3 & 3 & 3 & \\
\hline$f_{\mathrm{If}} O$ iajection & 2.62 & 350 & 318 & 232 & 264 & 213 & 190 & bek \\
\hline$f_{\text {If }} Q$ top courgy & 1.40 & 2.68 & 2.60 & 2.08 & 2.23 & 1.66 & 2.85 & MHs \\
\hline Top kimoic obery & 1857 & 1249 & 998 & 376 & 144 & 72 & 279 & MoVh \\
\hline tone/buncht & 19.9 & 8.32 & 7.06 & 8.7 & 5.64 & 4.12 & $1.2^{5}$ & $\times 10^{9}$ \\
\hline nocrolized enuinace & 8.8 & 6.0 & 3.4 & 3.9 & 3.0 & 2.3 & 22 & $x$ monemad \\
\hline stripping officy. $\odot s_{B}$ & - & 100 & 100 & 100 & 40 & 50 & so & $\$$ \\
\hline$Q$ afier $\mathrm{s}_{\mathrm{B}}$ & 1 & 8 & 14 & 29 & 53 & 77 & 90 & \\
\hline lonabunch after $S_{B}$ & 19.9 & 8.32 & 7.06 & 8.7 & 226 & 2.0 & 0.6 & $\times 10^{9}$ \\
\hline norm. emittance afier $S_{B}$ & 8.8 & 6.1 & 5.5 & 4.1 & 3.6 & 4.0 & 23 & $x$ mmenrad \\
\hline
\end{tabular}

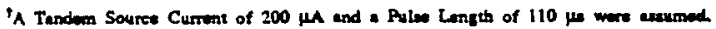

Requires soure developorme.

The normalized emittance after the stripping foil $S_{B}$ was calculated from a standard multiple Coulomb scattering formalism.

After extraction from the Booster, and on their way to the AGS $_{\text {f }}$ the ions pass through a stripping target. Experimental knowledge indicates that for atomic number $\leq 63$ the ions will be fully stripped with $100 \%$ efficiency. For ${ }^{127} \mathrm{I}$, the fully stripped state is achieved with $40 \%$ efficiency 8 on passing through foil $S_{B}$. The heavier ions such as ${ }^{197} \mathrm{Au}$ or ${ }^{238} \mathrm{U}$ are stripped to a filled $\mathrm{K}$-shell or two-electron atom. The stripping efficiency for ${ }^{238} \mathrm{U}$ in a charge $90^{+}$state is also known experimentally, ${ }^{9}$ and peaks at $50 \%$ for a kinetic energy of $220 \mathrm{MeV} / \mathrm{u}$. The stripping efficiency for 70 $\mathrm{MeV} / \mathrm{u}{ }^{197} \mathrm{Au}$ to a charge $77^{+}$state was deduced from theoretical work ${ }^{10}$ on ${ }^{208} \mathrm{~Pb}$ for the CERN SPS program. The result is considered reasonable, and will be known with more precision after a planned experiment ${ }^{11}$ at Berkeley in the fall of 1989.

The stripping in foil $S_{B}$ is accomplished with a foil of copper that, for gold, is $35 \mathrm{mg} / \mathrm{cm}^{2 \mathrm{p}}$ thick. If one does not ailow more than $10 \%$ growth in the emittance of $50 \pi \mathrm{mm} \cdot \mathrm{mrad}$. the stripper foil must be installed in a location where the lattice beta function does not exceed $2.6 \mathrm{~m}$ in both planes. This condition is accomplished using two pairs of quadrupole doublets.

\section{II.4 The AGS Component of the RHIC Injector Complex}

Inside the AGS all heavy ions with $A \leq 127$ will be fully stripped. Heavy ions such as ${ }^{197} \mathrm{Au}^{77+}$ and ${ }^{238} \mathrm{U}^{90+}$ will be accelerated with 2 electrons in a filled $\mathrm{K}$-shell.

The present vacuum inside the AGS is $10^{-7}$ Torr, with improvement to $10^{-8}$ Torr expected in the very near future. For an AGS vacuum pressure of $10^{-7}$ Torr, the beam losses of heavy ions with $A<100$ will be less than $1 \%$. This loss mechanism is mainly due to electron capture from the residual gas ions $\left(50 \% \mathrm{H}_{2}, 50 \%\right.$ $\mathrm{CO}$ ). For the heavier ions with two electrons in a filled $\mathrm{K}$-shell both electron knock-out and capture are possible. To estimate the depletion of these ions during the AGS acceleration cycle, the depletion rate $\lambda(t)$ was calculated from the formula,

$$
\lambda(t)=\beta(t) c \sum_{i} n_{i} \sigma_{i j}
$$

where $\beta(t)$ is the velocity of the ions, $n_{i}$ is the density of gas ions in the AGS, and $\sigma_{i g}$ is the interaction cross section for the accelerating ion on an individual gas species. $\sigma_{i g}$ is the sum of the knockout cross section $\sigma_{\mathrm{ig}}^{\mathrm{k}}$, and the capture cross section $\sigma_{\mathrm{ig}}^{\mathrm{f}}$. For the knock-out cross section we assume the theory of Bethe, ${ }^{128}$ which has been lested for Uranium by Gould et al. In this theory $\sigma_{i \mathrm{ig}}^{\mathrm{k}}$ is given by, ${ }^{3,12}$ 


$$
\sigma_{i}^{k}=4 \pi a_{0}^{2}\left(\frac{\alpha}{\beta}\right)^{2} \frac{R_{\infty}}{B_{\mathrm{a}}} Z_{\mathrm{i}}\left(1+Z_{\mathrm{i}}\right) f_{\mathrm{k}}\left\{\ell \mathrm{Ln}\left(\frac{2 \beta \gamma / \alpha R_{-}}{0.048 B_{\mathrm{a}}}\right)\right\}
$$

where $\alpha$ is the fine structure constant. $a_{0}$ the Bohr radius of the Hydrogen atom, $Z_{\mathrm{i}}$ the atomic number of the gas molecule, $R_{-}$the Rydberg constant, $B_{\mathrm{n}}$ the electron binding energy, and $f_{\mathrm{K}}$ an oscillator constant that takes the value 0.58 for ${ }^{238} \mathrm{U}$ in a charge $90^{+}$state. The kinematic factors. $\beta, \gamma$ are given by the acceleration cycle. The capture cross section (Radiative Electron Capture) into the $n^{\prime}$ th shell is given by ${ }^{5.13}$

$$
\sigma_{\mathrm{RBC}}=Z_{\mathrm{i}}\left((\gamma-1)+B_{\mathrm{q}} / m_{\mathrm{e}} c^{2}\right)^{2} \sigma(\varepsilon)\left(\gamma^{2}-1\right)
$$

where $m_{e}$ is the mass of the electron, and $B_{n}$ is the binding energy in the $n^{\prime}$ th shell. $\sigma(\varepsilon)$ is the photoionization cross section, which is tabulated for various shells in a range of nuclei, and $\varepsilon$ is the photon energy given by $\varepsilon=m_{e} c^{2}(\uparrow-1)+B_{n}$.

Figure II-3 shows the depletion of ions for a $0.6 \mathrm{sec}$ acceleration cycle. The depletion is defined by $D(t) \propto \exp \left(-\int_{0}^{t} \lambda\left(t^{\prime}\right) \mathrm{d} t^{\prime}\right)$, and two values of the AGS vacuum were assumed $\left(10^{-7}\right.$ Torr and $10^{-8}$ Torr). It can be seen that the beam depletion is only a few percent.

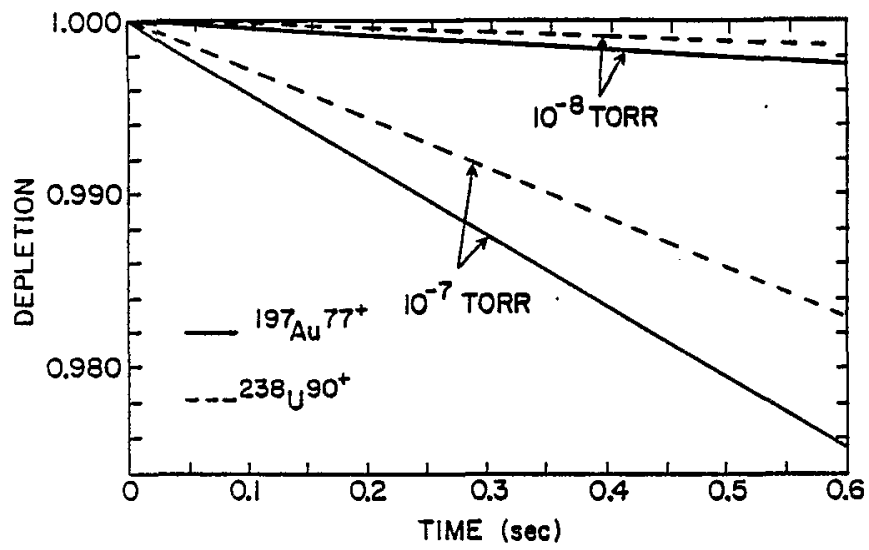

Fig. II-3. Depletion of beam in the AGS.

After acceleration in the AGS, the bunches are transferred into RHIC. For the heaviest ions, it is necessary 10 pass through the stripping foil $S_{A}$, to remove the last two electrons in the $\mathrm{K}$-shell. The position of this foil is shown schematically in Fig. II-2. At the top AGS energies $\left(10.4 \mathrm{GeV}\right.$ for ${ }^{197} \mathrm{Au}$ ), a design beta function value of $50 \mathrm{~m}$, and a rms scattering angle of $0.046 \mathrm{mrad}$ for a 100 $\mathrm{mg} / \mathrm{cm}^{2}$ copper foil, would correspond to an emittance growth of only $0.033 \pi \mathrm{mm} \cdot \mathrm{mrad}$. At these energies, negligible losses in particle numbers per bunch are expected during the final stripping process.

Taking into account the increase in the beam emittance due to the final stripping, the emittance values given in Table II-3 are just about those the beam would have when injected into the AGS. These emittances are considerably smaller than the ring betatron acceptance.

\section{Discussion}

The design criteria for RHIC demand that a range of species of ions be available for storage and acceleration. The wide variety of atomic properties associated with partially stripped heavy ions, together with the hardware constraints of the various preaccelerators of the injection complex severly limit the possible modes of injection into the collider. However, using properties of $\mathrm{K}$-shell atomic structure, the luminosity demands of RHIC can be readily met, and indeed the range of ions available for acceleration can be extended to include Uranium.

\section{References}

[ 1] "Conceptual Design of the Relativistic Heavy Ion Collider, RFIC." BNL 51432, May 1986.

[ 2] M.J. Rhondes-Brown and A.G. Ruggiero, "Source Current into the AGS. An analysis of the RHIC front end injection." RHIC Technical Note 32 (1988).

[ 3] P. Thieberger, M. McKeown and H.E. Wegner, IEEE Vol. NS-30, 2746 (1983).

[ 4] G.D. Alton, et al., to be published in Nuclear Instruments and Methods.

[ 5] M.J. Rhoades-Brown and H. Gould, "Accelerating Uranium in RHIC-11 Surviving the AGS Vacuum." RHIC Technical Note 38 (1988).

[ 6] M.J. Rhoades-Brown, "An Alternative Injection Scheme for Heavy Ions into RHIC." RHIC Technical Note 44 (1988).

[ 7 G. Young, "Multiple Coulomb Scattering and Emittance Growth in Stripper Foils," RHIC-PG-36 (1984).

[ 8] P. Thieberger H. Wegner, et al. IEEE Trans. NS-32, 1767 (1985).

[ 9] R. Anholt, et al. Phy. Rev. A36, 1586 (1987).

[10] H. Gould and B. Friedman, private communication.

[11] P. Thieberger, H. Wegner, M.J. Rhoades-Brown and Berkeley/Stanford experimentalists. Arrangements in progress at this time.

[12] H.E. Bethe, Ann. Phys. 5, 325 (1930).

[13] H. Gould et al., Phys. Rev. Lett. 52, 180 (1984). 